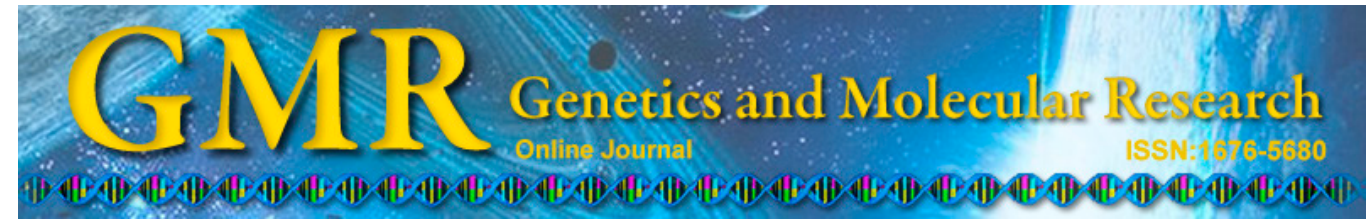

\title{
Mitochondrial DNA haplogroups and somatic mutations are associated with lung cancer in patients from Southwest China
}

\author{
Y. Fang ${ }^{1 *}$, H.Y. Yang ${ }^{2 *}$, Y.H. Shi ${ }^{3}$, J.H. Cui ${ }^{4}$, L.Y. $\mathrm{Li}^{5}$, Y.C. $\mathrm{Xu}^{5}$ and \\ J.L. Shao ${ }^{1}$ \\ ${ }^{1}$ Department of Anesthesiology, \\ The First Affiliated Hospital of Kunming Medical University, \\ Kunming, Yunnan Province, China \\ ${ }^{2}$ Kunming Maternal and Child Health Center, Kunming, \\ Yunnan Province, China \\ ${ }^{3}$ Clinical Laboratory Department, Cancer Hospital of Shanxi, \\ Taiyuan, Shanxi, China \\ ${ }^{4}$ Department of Medical Service, PLA Unit 65176, Dalian, Liaoning, China \\ ${ }^{5}$ Department of Cardiology and Clinical Laboratory Department, \\ 211th Hospital of the PLA, Harbin, Heilongjiang, China \\ *These authors contributed equally to this study. \\ Corresponding author: J.L. Shao \\ E-mail: jianlinshaoydyy@163.com
}

Genet. Mol. Res. 14 (2): 5031-5043 (2015)

Received June 23, 2014

Accepted October 9, 2014

Published May 12, 2015

DOI http://dx.doi.org/10.4238/2015.May.12.6

\begin{abstract}
Mitochondrial DNA mutations play crucial roles in the pathogenesis and progression of human malignancies. Therefore, to determine whether maternal background or mitochondrial DNA somatic mutations were essential cofactors in the lung cancer of Chinese patients as well, the complete mitochondrial DNA displacement loop of the primary cancerous, matched para-cancerous normal and distant normal tissues for 79 Chinese patients with lung cancer were analyzed in this study. Our results indicated that the higher detected frequency of haplogroups prevalent in southern East Asia $(53.16 \%$; 42/79) versus
\end{abstract}


those of northern East Asia in the studied population supported the southern East Asian characteristics of the Chinese lung cancer group. Further statistical analysis revealed that the haplogroups $\mathrm{F}^{*}$ and $\mathrm{G}^{*}$ contributed to the susceptibility to lung cancer in Chinese patients. In addition, by comparing sequences from different tissues of the same patients, a total of eight somatic mutations from six patients were detected. Combined with the fourteen somatic mutations identified in our previous study, the somatic mutation spectrum of the 79 Chinese patients with lung cancer was $25.32 \%$ (20/79). Our results suggest that mitochondrial DNA haplogroups and somatic mutations are associated with lung cancer in patients from Yunnan, Southwest China, and that somatic mitochondrial DNA mutations in the displacement loop can serve as potential biomarkers for clinical utility.

Key words: Lung cancer; Mitochondrial DNA; Haplogroup; Somatic mutation

\section{INTRODUCTION}

Lung cancer is the leading cause of cancer deaths globally, accounting for $13 \%$ of all deaths (Yang Ai et al., 2013) and 30\% of all cancer-related deaths (Ferlay et al., 2010; Cao et al., 2011). Lung cancer has also caused approximately 400,000 deaths annually in China (Yang et al., 2009), with late diagnosis and suboptimal therapies being considered the main cause of the low 5-year survival rate in lung cancer.

Mitochondria serve as the crucial intracellular organelle responsible for regulating cellular energy metabolism, producing free radicals, and initiating and executing apoptotic pathways (Wallace, 1995). Much evidence has supported the association between mitochondrial DNA (mtDNA) mutations and various diseases, with somatic mutations in particular having been correlated with various tumors (Wallace, 2005).

Maternally inherited variations in mtDNA have been considered the result of adaptations in the ancestors of modern humans to habitation in cold climatic environments (Mishmar et al., 2003; Ruiz-Pesini et al., 2004). They have also been associated with bioenergetic or mitochondrial dysfunction (Mishmar et al., 2003; Ruiz-Pesini et al., 2004; Wallace, 2005), with previous studies demonstrating associations between mutations of haplogroup N9a and type 2 diabetes in Asians (Fuku et al., 2007) and between haplogroups M7b1'2 and M8 with the expression of Leber hereditary optic neuropathy in Chinese families with the m11778G/A mutation (Ji et al., 2008). mtDNA is highly susceptible to mutation because of its continuous exposure to high levels of reactive oxygen species generated during oxidative phosphorylation (Wallace et al., 1999), leading to a higher mutation rate of mtDNA than of nuclear DNA (Yakes and Van Houten, 1997). Such mutations, which occur in individual cells are not inherited from a parent, and are not passed on to offspring, are called somatic mutations. Furthermore, given the paucity of spacer regions between human mitochondrial genes, a mutation in the mtDNA will most likely involve a functionally important region of the mitochondrial genome. Increasing numbers of studies have revealed that somatic mtDNA mutation may be involved in carcinogenesis and tumor progression. These mutations have been detected with relatively higher frequency by comparison between the primary cancerous, matched para-cancerous 
normal, and distant normal tissues from the same patients (Brandon et al., 2006; Chatterjee et al., 2006). To date, a large number of somatic mutations have been detected among various kinds of tumor tissues, such as in breast cancer (Wang et al., 2007; Alhomidi et al., 2013), gastric cancer (Hung et al., 2010), esophageal squamous cell carcinoma (Kumimoto et al., 2004), lung cancer (Jin et al., 2007; Wang and Zhao, 2011; Fang et al., 2013; Yang Ai et al., 2013), and in aging individuals (Williams et al., 2013). Our previous study also revealed elevated somatic mutation rates through the examination of thirty entire mtDNA genomes from ten Chinese patients with lung cancer (Fang et al., 2013), as well as the poly-C repeat stretch (D310) of 79 patients (Chen et al., 2014). Therefore, both germline and somatic mtDNA mutations have been deemed to play crucial roles in tumorigenesis.

Human mtDNA is a circular, double-stranded molecule comprised of approximately $16,569 \mathrm{bp}$. It contains a displacement loop (D-loop) and 37 genes, coding for $12 \mathrm{~S}$ and $16 \mathrm{~S}$ rRNAs, 22 tRNAs, and 13 polypeptides (Anderson et al., 1981; Andrews et al., 1999). The mtDNA D-loop, a 1124-bp long fragment (from position 16,024 to 576) (NC_012920) (Andrews et al., 1999), is a non-coding region, and acts as a promoter for both the heavy and light strands of the mtDNA, containing essential transcription and replication elements. It also contains two hypervariable regions (HVI at position 16,024 to 16,383; and HVII at 57 to 372) (NC_012920) (Andrews et al., 1999), which have consequently been widely used in forensic analyses and medical diagnosis (Sharma et al., 2005; Li et al., 2012). Therefore, we speculated that the variation of the mtDNA D-loop region in Chinese patients with lung cancer would better illustrate the crucial role that mtDNA might play in the mechanism of lung carcinogenesis.

In this study, to determine whether the maternal haplogroups or mtDNA somatic mutations played crucial roles in Chinese patients with lung cancer, both the germ line and somatic mutations of the mtDNA D-loop were identified in 237 samples from 79 Chinese patients with lung cancer; our previously reported D310 mutations were also analyzed.

\section{MATERIAL AND METHODS}

\section{Tissue specimens}

A total of 237 tissue samples, including the primary lung cancerous tissue, corresponding para-cancerous normal tissue, and distant normal tissue, were collected from 79 patients with lung cancer who received treatment at the First Affiliated Hospital of Kunming Medical University or the Second People's Hospital of Yunnan Province between 2011 and 2012. The primary cancerous and para-cancerous normal tissues were sampled by manual microdissection from hematoxylin and eosin-stained slides. The different tissues from the same individual were marked with suffix "A", "B", or "C", respectively; "A" referred to the paracancerous normal tissue, "B" referred to the primary lung cancerous tissue, and " $\mathrm{C}$ " referred to the distant normal tissue (blood). All procedures were supervised and approved by the human tissue research committee of the First Affiliated Hospital of Kunming Medical University, and informed consents were obtained from all participants.

\section{DNA extraction, polymerase chain reaction (PCR) amplification and sequence analysis}

The genomic DNA was extracted using standard phenol/chloroform methodology, and stored at $-20^{\circ} \mathrm{C}$ for future use. The mtDNA D-loop regions (spanning nucleotide posi- 
tions 16024-16569/1-576) were amplified and sequenced as fully described in Zhao et al. (2009). Mutations were recorded by comparison with the revised Cambridge reference sequence (rCRS) (Andrews et al., 1999). All subjects were allocated into a specific haplogroup based on their control-region information according to the updated worldwide phylogenetic tree constructed with the entire mtDNA genome (van Oven and Kayser, 2009). Principle component analysis (PCA) was conducted as described previously (Yao et al., 2002a) by taking the haplogroup frequencies of the lung cancer group and of healthy populations as input factors. Statistical analysis was conducted using the SPSS 13.0 software package (SPSS, Chicago, IL, USA), with $\mathrm{P}<0.05$ as a statistically significant difference.

\section{Cloning and sequencing of mtDNA somatic mutations}

Cloning and sequencing analysis were performed to verify the authenticity of the somatic mutations detected in the mtDNA D-loop region among Chinese patients with lung cancer. The PCR products of the newly extracted DNA from primary lung cancerous tissues and distant normal tissues in patients with somatic mutations were purified and transferred to the pUC18 vector. The clones were selected and sequenced directly with forward and reverse vector primers, and the sequences were compared with the rCRS (Andrews et al., 1999).

\section{RESULTS}

As shown in Table 1, all the lung cancer patients investigated from Southwest China were allocated to haplogroups prevalent in East Asia, Southeast Asia, West Eurasia, or South Asia. The East and Southeast Asian prevalent haplogroups accounted for a significantly higher proportion $(96.20 \%$; 76/79) of the patients than did the West Eurasian and South Asian predominant haplogroups (2.53\% and $1.27 \%$, respectively). Among the former, the southern East Asian and Southeast Asian prevalent haplogroups, such as B, F, M7, M9, M71, M*, R9, and R11 (Wen et al., 2004a; Kong et al., 2011), accounting for 50.63\% (40/79) of the East and Southeast Asian prevalent haplogroups, which was more than the northern East Asian prevalent haplogroups, such as A, C, Z, D, G and N9, which accounted for $44.30 \%(35 / 79)$ of the patients.

To evaluate the association of mtDNA haplogroups with the lung cancer population, the mtDNA variations of 490 healthy individuals from 11 Yunnan ethnic and Han populations were retrieved from the literature (Yao et al., 2002b; Wen et al., 2004a,b, 2005; Zhao et al., 2009). As PCA has been shown to be a powerful tool for detecting true associations in mitochondrial medical genetics (Biffi et al., 2010), we constructed a PCA map by taking the haplogroup frequencies of 79 lung cancer patients and 490 healthy individuals from 11 ethnic and Han groups as input factors. Our results showed that the lung cancer group was clustered with the Naxi, Pumi, and Hani ethnic groups from Yunnan Province. They did not show any separated cluster pattern, as shown in Figure 1, supporting that there was no essential difference between them, and excluding the possibility of population stratification between the lung cancer population and other 11 control groups (including 1 Han and 10 ethnic groups). Further, to confirm whether there was any specific mtDNA haplogroup associated with lung cancer, the statistically significant differences were estimated between the mtDNA haplogroup frequencies of the lung cancer group and healthy populations by pooling the 1 Han Chinese and 10 ethnic groups from different regions of Yunnan as control population for analysis in order to avoid the biasing of results because of small sample size and sampling location. 
Table 1. mtDNA mutations in 237 samples from 79 patients with lung cancer from Yunnan, Southwest China.

\begin{tabular}{|c|c|c|c|c|}
\hline Sample & Haplogroups & HVSI (16000+) & HVSII & Readable region \\
\hline $1 \mathrm{~A}$ & M7 & 129223297 & $73150159199263315+C 489$ & $16013-16569 / 1-554$ \\
\hline 1B & M7 & 129223297 & $73150159199263315+C 489$ & $16012-16569 / 1-549$ \\
\hline $1 \mathrm{C}$ & M7 & 129223297 & $73150159199263315+C 489$ & $16018-16569 / 1-590$ \\
\hline $2 \mathrm{~A}$ & D4 & 223362 & $73152195263315+C 489$ & $16013-16569 / 1-531$ \\
\hline 2B & D4 & 223362 & $73152195263315+C 489$ & $16013-16569 / 1-588$ \\
\hline $2 \mathrm{C}$ & D4 & 223362 & $73152195263315+C 489$ & $16012-16569 / 1-587$ \\
\hline $3 \mathrm{~A}$ & $\mathrm{D} 4 \mathrm{e}$ & 092223362 & $7394263309+\mathrm{C} 315+\mathrm{C} 489$ & $16012-16569 / 1-542$ \\
\hline 3B & $\mathrm{D} 4 \mathrm{e}$ & 092223362 & $7394263309+\mathrm{C} 315+\mathrm{C} 489$ & $16014-16569 / 1-588$ \\
\hline $3 \mathrm{C}$ & $\mathrm{D} 4 \mathrm{e}$ & 092223362 & 7394263 309+C $315+C 489$ & $16013-16569 / 1-588$ \\
\hline $4 \mathrm{~A}$ & F1 & 189304519 & 73146 249d 263 309+C 315+C 522-523d & $16016-16569 / 1-542$ \\
\hline 4B & $\mathrm{F} 1$ & 189304519 & $73146249 \mathrm{~d} 263$ 309+C 315+C 522-523d & $16012-16569 / 1-587$ \\
\hline $4 \mathrm{C}$ & $\mathrm{F} 1$ & 189304519 & 73146249 d 263 309+C 315+C 522-523d & $16012-16569 / 1-549$ \\
\hline $5 \mathrm{~A}$ & $\mathrm{~F}$ & 260298355362 & 73 249d 263 309+C 315+C 709 & $16016-16569 / 1-717$ \\
\hline $5 \mathrm{~B}$ & $\mathrm{~F}$ & 260298355362 & 73 249d 263 309+C 315+C & $16016-16569 / 1-614$ \\
\hline $5 \mathrm{C}$ & $\mathrm{F}$ & 260298355362 & 73 249d 263 309+C 315+C & $16013-16569 / 1-499$ \\
\hline $6 \mathrm{~A}$ & $\mathrm{D} 4 \mathrm{a}$ & 129223362519 & $73152217263309+2 C$ 315+C 489 & $16016-16569 / 1-543$ \\
\hline $6 \mathrm{~B}$ & D4a & 129223362519 & $73152217263309+2 C$ 315+C 489 & $16013-16569 / 1-560$ \\
\hline $6 \mathrm{C}$ & $\mathrm{D} 4 \mathrm{a}$ & 129223362519 & $73152217263309+2 \mathrm{C} 315+\mathrm{C} 489$ & $16012-16569 / 1-554$ \\
\hline 7A & $\mathrm{B} 4 \mathrm{c}$ & $147182+\mathrm{C} 189217235519$ & $73146263 \mathbf{3 0 9 + 2 C} 315+\mathrm{C}$ & $16014-16569 / 1-581$ \\
\hline 7B & $\mathrm{B} 4 \mathrm{c}$ & $147182+\mathrm{C} 189217235519$ & $73146263 \mathbf{3 0 9}+\mathrm{C} 315+\mathrm{C}$ & $16017-16569 / 1-562$ \\
\hline 7C & $\mathrm{B} 4 \mathrm{c}$ & $147182+\mathrm{C} 189217235519$ & $73146263 \mathbf{3 0 9}+\mathbf{2 C} 315+\mathrm{C}$ & $16017-16569 / 1-563$ \\
\hline $8 \mathrm{~A}$ & F1ala & 129162172304311519 & 73152 249d $263315+C 477$ 522-523d & $16011-16569 / 1-598$ \\
\hline $8 \mathrm{~B}$ & F1ala & 129162172304311519 & 73152 249d 263 315+C 477 522-523d & $16012-16569 / 1-588$ \\
\hline $8 \mathrm{C}$ & F1ala & 129162172304311519 & 73152 249d 263 315+C 477 522-523d & $16013-16569 / 1-587$ \\
\hline 9A & $\mathrm{D}$ & 172223362 & $73263309+\mathrm{C} 315+\mathrm{C} 489$ & $16012-16569 / 1-585$ \\
\hline 9B & $\mathrm{D}$ & 172223362 & $73263309+\mathrm{C} 315+\mathrm{C} 489$ & $16009-16569 / 1-588$ \\
\hline 9C & $\mathrm{D}$ & 172223362 & $73263309+\mathrm{C} 315+\mathrm{C} 489$ & $16012-16569 / 1-580$ \\
\hline $10 \mathrm{~A}$ & $\mathrm{R} 9 \mathrm{~b}$ & 192304309390519 & $73152263309+\mathrm{C} 315+\mathrm{C}$ & $16015-16569 / 1-553$ \\
\hline 10B & $\mathrm{R} 9 \mathrm{~b}$ & 192304309390519 & $73152263309+\mathrm{C} 315+\mathrm{C}$ & $16012-16569 / 1-555$ \\
\hline $10 \mathrm{C}$ & $\mathrm{R} 9 \mathrm{~b}$ & 192304309390519 & $73152263309+\mathrm{C} 315+\mathrm{C}$ & $16018-16569 / 1-552$ \\
\hline $11 \mathrm{~A}$ & $\mathrm{~B} 4 \mathrm{a}$ & 181C 182C 183C 189213217261519 & 61A $6273263315+C 522-523 d$ & $16012-16569 / 1-572$ \\
\hline 11B & $\mathrm{B} 4 \mathrm{a}$ & 181C 182C 183C 189213217261519 & 61 A $6273263315+C 522-523 d$ & $16013-16569 / 1-590$ \\
\hline $11 \mathrm{C}$ & $\mathrm{B} 4 \mathrm{a}$ & 181C 182C 183C 189213217261519 & $61 \mathrm{~A} 6273263315+\mathrm{C} 522-523 \mathrm{~d}$ & $16013-16569 / 1-589$ \\
\hline $12 \mathrm{~A}$ & $M^{*}$ & $129223287311327 \mathrm{~A}$ & 647393189200263 309+2C 315+C 485489 & $16012-16569 / 1-588$ \\
\hline 12B & $\mathrm{M}^{*}$ & $129223287311327 \mathrm{~A}$ & 647393189200263 309+C 315+C 485489 & $16013-16569 / 1-555$ \\
\hline $12 \mathrm{C}$ & $M^{*}$ & $129223287311327 \mathrm{~A}$ & $647393189200263 \mathbf{3 0 9 + 2 C} 315+C 485489$ & $16014-16569 / 1-553$ \\
\hline $13 \mathrm{~A}$ & N9a & 129162223250257 A 261 & $73150263309+\mathrm{C} 315+\mathrm{C}$ & $16018-16569 / 1-555$ \\
\hline 13B & N9a & 129162223250257 A 261 & $73150263309+\mathrm{C} 315+\mathrm{C}$ & $16013-16569 / 1-553$ \\
\hline $13 \mathrm{C}$ & N9a & 129162223250 257A 261 & $73150263309+\mathrm{C} 315+\mathrm{C}$ & $16012-16569 / 1-450$ \\
\hline $14 \mathrm{~A}$ & D5 & $183 \mathrm{C} 189362519$ & $73150152263309+C 315+C 456489$ & $16014-16569 / 1-552$ \\
\hline 14B & D5 & 183C 189362519 & $73150152263309+C$ 315+C 456489 & $16015-16569 / 1-554$ \\
\hline $14 \mathrm{C}$ & D5 & $183 \mathrm{C} 189362519$ & $73150152263309+C \quad 315+C 456489$ & $16015-16569 / 1-550$ \\
\hline $15 \mathrm{~A}$ & D5 & $092148183 \mathrm{C} 189362519$ & $73150152185263315+C 456489522-523 d$ & $16013-16569 / 1-552$ \\
\hline 15B & D5 & $092148183 \mathrm{C} 189362519$ & $73150152185263315+C 456489522-523 d$ & $16013-16569 / 1-555$ \\
\hline $15 \mathrm{C}$ & D5 & $092148183 \mathrm{C} 189362519$ & $73150152185263315+C 456489522-523 d$ & $16013-16569 / 1-550$ \\
\hline $16 \mathrm{~A}$ & $\mathrm{~F} 1$ & 129172304519 & 73 249d 263 309+2C 315+C $466520-524 d$ & $16007-16569 / 1-586$ \\
\hline 16B & $\mathrm{F} 1$ & 129172304519 & 73 249d 263 294h $309+2$ C $315+C 466$ 520-524d & $16012-16569 / 1-586$ \\
\hline $16 \mathrm{C}$ & F1 & 129172304519 & 73 249d 263 309+2C 315+C 466 520-524d & $16016-16569-1-543$ \\
\hline 17A & M7 & $129192223297301 G 391519$ & $73150199263309+C$ 315+C 489 & $16017-16569 / 1-572$ \\
\hline 17B & M7 & $129192223297301 G 391519$ & $73150199263309+C$ 315+C 489 & $16016-16569 / 1-544$ \\
\hline $17 \mathrm{C}$ & M7 & $129192223297301 G 391519$ & $73150199263309+C 315+C 489$ & $16020-61569 / 1-573$ \\
\hline $18 \mathrm{~A}$ & A & 223274290319362519527 & $73152235263315+C 456522-523 d$ & $16015-16569 / 1-591$ \\
\hline 18B & A & 223274290319362519527 & $73152235263315+C 456522-523 d$ & $16009-16569 / 1-549$ \\
\hline $18 \mathrm{C}$ & A & 223274290319362519527 & $73152235263315+C 456522-523 d 663750$ & $16012-16569 / 1-778$ \\
\hline 19A & F1a & 129162172274304519 & 73 249d 263 315+C 522-523d 548 & $16016-16569 / 1-555$ \\
\hline 19B & F1a & 129162172274304519 & 73 249d 263 315+C 522-523d 548 & $16012-16569 / 1-620$ \\
\hline $19 \mathrm{C}$ & F1a & 129162172274304519 & 73 249d $263315+C$ 522-523d 548 & $16012-16569 / 1-550$ \\
\hline $20 \mathrm{~A}$ & A & 223235290311319362519 & 73152234235263 309+2C $315+C$ 522-523d & $16007-16569 / 1-555$ \\
\hline 20B & A & 223235290311319362519 & 73152234235263 309+C 315+C 522-523d 663 & $16017-16569 / 1-667$ \\
\hline $20 \mathrm{C}$ & A & 223235290311319362519 & 73152234235263 309+2C 315+C 522-523d & $16017-16569 / 1-575$ \\
\hline $21 \mathrm{~A}$ & B4 & 182C 183C 189217223519 & 73146185189195263 309+2C 315+C & $16011-16569 / 1-322$ \\
\hline 21B & B4 & 182C 183C 189217223519 & $73146185189195263309+2$ C $315+C 513$ & $16016-16569 / 1-555$ \\
\hline
\end{tabular}

Continued on next page 


\begin{tabular}{|c|c|c|c|c|}
\hline Sample & Haplogroups & HVSI (16000+) & HVSII & Readable region \\
\hline $21 \mathrm{C}$ & B4 & 182C 183C 189217223519 & $73146185189195263309+2 C 315+C$ & $16008-16569 / 1-323$ \\
\hline $22 \mathrm{~A}$ & M71 & 223269271311 & $73150151263 \mathbf{3 0 9 + 2 C} 315+\mathrm{C} 489$ & $16013-16569 / 1-544$ \\
\hline $22 \mathrm{~B}$ & M71 & 223269271311 & $73150151263 \mathbf{3 0 9 + C} 315+\mathrm{C} 489$ & $16012-16569 / 1-588$ \\
\hline $22 \mathrm{C}$ & M71 & 223269271311 & $73150151263 \mathbf{3 0 9 + 2 C} 315+C 489$ & $16010-16569 / 1-563$ \\
\hline $23 \mathrm{~A}$ & $\mathrm{~F}$ & 304 & 73249 d $263309+\mathrm{C} 315+\mathrm{C}$ & $16013-16569 / 1-563$ \\
\hline $23 \mathrm{~B}$ & $\mathrm{~F}$ & 304 & 73249 d $263309+C$ 315+C & $16013-16569 / 1-546$ \\
\hline $23 \mathrm{C}$ & $\mathrm{F}$ & 304 & $73249 \mathrm{~d} 263309+\mathrm{C} 315+\mathrm{C}$ & $16009-16569 / 1-570$ \\
\hline $24 \mathrm{~A}$ & $\mathrm{C}$ & 223298327362519 & 73249 d $263309+C \quad 315+C 489$ & $16010-16569 / 1-554$ \\
\hline $24 \mathrm{~B}$ & $\mathrm{C}$ & 223298327362519 & 73249 d $263309+C \quad 315+C 489$ & $16009-16569 / 1-392$ \\
\hline $24 \mathrm{C}$ & $\mathrm{C}$ & 223298327362519 & 73249 d $263309+C \quad 315+C 489$ & $16008-16569 / 1-565$ \\
\hline $25 \mathrm{~A}$ & $\mathrm{H}$ & 519 & $263309+\mathrm{C} 315+\mathrm{C}$ & $16012-16569 / 1-554$ \\
\hline $25 \mathrm{~B}$ & $\mathrm{H}$ & 519 & $263309+2 C 315+C$ & $16012-16569 / 1-566$ \\
\hline $25 \mathrm{C}$ & $\mathrm{H}$ & 519 & $263309+\mathrm{C} 315+\mathrm{C}$ & $16012-16569 / 1-542$ \\
\hline $26 \mathrm{~A}$ & $\mathrm{~A}$ & 223290319 & $73152235263309+2 C 315+C \quad 522-523 d$ & $16013-16569 / 1-549$ \\
\hline $26 \mathrm{~B}$ & $\mathrm{~A}$ & 223290319 & $73152235263309+2 \mathrm{C} 315+\mathrm{C}$ & $16011-16569 / 1-506$ \\
\hline $26 \mathrm{C}$ & A & 223290319 & $73152235263309+2 C 315+C \quad 522-523 d$ & $16012-16569 / 1-553$ \\
\hline $27 \mathrm{~A}$ & F1a & 108129162172304519 & $73249 d 263315+C$ 522-523d & $16008-16569 / 1-588$ \\
\hline $27 \mathrm{~B}$ & F1a & 108129162172304519 & $73249 \mathrm{~d} 263315+\mathrm{C} 522-523 \mathrm{~d}$ & $16012-16569 / 1-554$ \\
\hline $27 \mathrm{C}$ & F1a & 108129162172304519 & $73249 \mathrm{~d} 263315+\mathrm{C} 522-523 \mathrm{~d}$ & $16012-16569 / 1-555$ \\
\hline $28 \mathrm{~A}$ & $\mathrm{U}$ & 051126178179234247 & $73146152263315+C 522-523 d$ & $16009-16569 / 1-543$ \\
\hline $28 \mathrm{~B}$ & $\mathrm{U}$ & 051126178179234247 & $73146152263315+C 522-523 d$ & $16013-16569 / 1-556$ \\
\hline $28 \mathrm{C}$ & $\mathrm{U}$ & 051126178179234247 & $73146152263315+C 522-523 d$ & $16014-16569 / 1-547$ \\
\hline $29 \mathrm{~A}$ & $\mathrm{~B} 4 \mathrm{a}$ & $093182 \mathrm{C} 183 \mathrm{C} 189217261519$ & $73146204263 \mathbf{3 0 9}+\mathrm{C} 315+\mathrm{C}$ & $16007-16569 / 1-316$ \\
\hline $29 \mathrm{~B}$ & B4a & $093182 \mathrm{C} 183 \mathrm{C} 189217261519$ & $73146204263 \mathbf{3 0 9 + 2 C} 315+C$ 522-523d 709 & $16013-16569 / 1-743$ \\
\hline $29 \mathrm{C}$ & $\mathrm{B} 4 \mathrm{a}$ & $093182 \mathrm{C} 183 \mathrm{C} 189217261519$ & $73146204263 \mathbf{3 0 9}+\mathrm{C} 315+\mathrm{C}$ & $16013-16569 / 1-319$ \\
\hline $30 \mathrm{~A}$ & $\mathrm{G}$ & 184223290362519 & $73143263315+C 489$ & $16014-16569 / 1-584$ \\
\hline $30 \mathrm{~B}$ & G & 184223290362519 & $73143263 \mathbf{3 0 9}+\mathbf{C} 315+\mathrm{C} 489$ & $16015-16569 / 1-590$ \\
\hline $30 \mathrm{C}$ & G & 184223290362519 & $73143263315+C 489$ & $16132-16569 / 1-573$ \\
\hline $31 \mathrm{~A}$ & F1a & 129172304519 & $73249 \mathrm{~d} 263309+2 \mathrm{C} 315+\mathrm{C} 522-523 d$ & $16014-16569 / 1-551$ \\
\hline $31 \mathrm{~B}$ & F1a & 129172304519 & $73249 \mathrm{~d} 263309+2 \mathrm{C} 315+\mathrm{C} 522-523 \mathrm{~d}$ & $16018-16569 / 1-584$ \\
\hline $31 \mathrm{C}$ & F1a & 129172304519 & $73249 \mathrm{~d} 263309+2 \mathrm{C} 315+\mathrm{C}$ & $16013-16569 / 1-365$ \\
\hline $32 \mathrm{~A}$ & R11 & $111172183 C 189223362519$ & $73185189195234263 \mathbf{3 0 9}+\mathrm{C} 315+\mathrm{C} 522-523 \mathrm{~d}$ & $16017-16569 / 1-550$ \\
\hline $32 \mathrm{~B}$ & $\mathrm{R} 11$ & $111172183 \mathrm{C} 189223362519$ & $73185189195234263 \mathbf{3 0 9}+\mathrm{C} 315+\mathrm{C} 522-523 d$ & $16016-16569 / 1-543$ \\
\hline $32 \mathrm{C}$ & R11 & $111172183 \mathrm{C} 189223362519$ & $73185189195234263 \mathbf{3 0 9}+\mathbf{3 C} 315+\mathrm{C}$ & $16012-16569 / 1-491$ \\
\hline $33 \mathrm{~A}$ & A & 223290319362 & $73151152200235263309+C \quad 315+C 522-523 d$ & $16012-16569 / 1-554$ \\
\hline $33 \mathrm{~B}$ & $\mathrm{~A}$ & 223290319362 & $73151152200235263309+C \quad 315+C \quad 522-523 d$ & $16013-16569 / 1-600$ \\
\hline $33 \mathrm{C}$ & A & 223290319362 & $73151152200235263309+C \quad 315+C \quad 522-523 d$ & $16013-16569 / 1-588$ \\
\hline $34 \mathrm{~A}$ & D4 & 124223362519 & $73194263309+\mathrm{C} 315+\mathrm{C} 489522-523 \mathrm{~d}$ & $16016-16569 / 1-573$ \\
\hline $34 \mathrm{~B}$ & D4 & 124223362519 & $73194263309+\mathrm{C} 315+\mathrm{C}$ & $16021-16569 / 1-414$ \\
\hline $34 \mathrm{C}$ & D4 & 124223362519 & $73194263309+C \quad 315+C 489$ 522-523d & $16021-16569 / 1-572$ \\
\hline $35 \mathrm{~A}$ & A & 223290319362 & $73151152200235263315+\mathrm{C} 522-523 d$ & $16012-16569 / 1-599$ \\
\hline $35 \mathrm{~B}$ & $\mathrm{~A}$ & 223290319362 & $73151152200235263315+C$ 522-523d & $16015-16569 / 1-544$ \\
\hline $35 \mathrm{C}$ & A & 223290319362 & $73151152200235263315+C$ 522-523d & $16013-16569 / 1-587$ \\
\hline $36 \mathrm{~A}$ & B4 & $182 \mathrm{C} 183 \mathrm{C} 189217261357519$ & $73263309+\mathrm{C} 315+\mathrm{C}$ & $16013-16569 / 1-438$ \\
\hline $36 \mathrm{~B}$ & B4 & $182 \mathrm{C} 183 \mathrm{C} 189217261357519$ & $73263309+\mathrm{C} 315+\mathrm{C} 522-523 d$ & $16014-16569 / 1-586$ \\
\hline $36 \mathrm{C}$ & B4 & $182 \mathrm{C} 183 \mathrm{C} 189217261357519$ & $73263309+\mathrm{C} 315+\mathrm{C} 522-523 \mathrm{~d}$ & $16017-16569 / 1-587$ \\
\hline $37 \mathrm{~A}$ & $\mathrm{D}$ & $164172182 \mathrm{C} 183 \mathrm{C} 189223266362$ & $73150263309+C \quad 315+C 489522-523 d$ & $16017-16569 / 1-588$ \\
\hline $37 \mathrm{~B}$ & $\mathrm{D}$ & $164172182 \mathrm{C} 183 \mathrm{C} 189223266362$ & $73150263309+\mathrm{C} 315+\mathrm{C} 489522-523 d$ & $16013-16569 / 1-587$ \\
\hline $37 \mathrm{C}$ & $\mathrm{D}$ & $164172182 \mathrm{C} 183 \mathrm{C} 189223266362$ & $73150263309+\mathrm{C} 315+\mathrm{C} 489$ & $16009-16569 / 1-520$ \\
\hline $38 \mathrm{~A}$ & N9a & 129223 257A 261519 & $73150263309+\mathrm{C} 315+\mathrm{C}$ & $16012-16569 / 1-588$ \\
\hline $38 \mathrm{~A}$ & $\mathrm{N9a}$ & $129223257 \mathrm{~A} 261519$ & $73150263309+\mathrm{C} 315+\mathrm{C}$ & $16010-16569 / 1-584$ \\
\hline $38 \mathrm{C}$ & N9a & $129223257 \mathrm{~A} 261519$ & $73150263309+\mathrm{C} 315+\mathrm{C}$ & $16013-16569 / 1-582$ \\
\hline $39 \mathrm{~A}$ & $\mathrm{Z}$ & 093h 185223260271298311390 & $73152249 d 263315+C \quad 319489$ & $16014-16569 / 1-588$ \\
\hline $39 \mathrm{~B}$ & $\mathrm{Z}$ & 093h 185223260271298311390 & 73152249 d $263315+C \quad 319489$ & $16014-16569 / 1-587$ \\
\hline $39 \mathrm{C}$ & $\mathrm{Z}$ & 093185223260271298311390 & 73152249 d $263315+C \quad 319489$ & $16014-16569 / 1-578$ \\
\hline $40 \mathrm{~A}$ & A & 086223290319362 & $73152235263 \mathbf{3 0 9 + 2 C} 315+\mathrm{C} 522-523$ d 538 & $16012-16569 / 1-553$ \\
\hline 40B & A & 086223290319362 & $73152235263 \mathbf{3 0 9}+\mathrm{C} 315+\mathrm{C} 522-523 \mathrm{~d} 538$ & $16016-16569 / 1-555$ \\
\hline $40 \mathrm{C}$ & A & 086223290319362 & $73152235263 \mathbf{3 0 9 + 2 C} 315+\mathrm{C} 522-523$ d 538 & $16010-16569 / 1-585$ \\
\hline $41 \mathrm{~A}$ & $\mathrm{C}$ & 223298327519 & $73249 \mathrm{~d} 263309+\mathrm{C} 315+\mathrm{C} 489$ & $16013-16569 / 1-539$ \\
\hline $41 \mathrm{~B}$ & $\mathrm{C}$ & 223298327519 & $73249 \mathrm{~d} 263309+\mathrm{C} 315+\mathrm{C} 489$ & $16012-16569 / 1-562$ \\
\hline $41 \mathrm{C}$ & $\mathrm{C}$ & 223298327519 & $73249 \mathrm{~d} 263309+\mathrm{C} 315+\mathrm{C} 489$ & $16000-16569 / 1-573$ \\
\hline $42 \mathrm{~A}$ & $\mathrm{D} / \mathrm{G}$ & 223311362519 & $73150194263315+C 489522-523 d$ & $16012-16569 / 1-543$ \\
\hline
\end{tabular}

Continued on next page 


\begin{tabular}{|c|c|c|c|c|}
\hline Sample & Haplogroups & HVSI (16000+) & HVSII & Readable region \\
\hline$\overline{42 B}$ & $\mathrm{D} / \mathrm{G}$ & 223311362519 & $73150194263315+C 489522-523 d$ & $16013-16569 / 1-581$ \\
\hline $42 \mathrm{C}$ & $\mathrm{D} / \mathrm{G}$ & 223311362519 & $73150194263315+C 489522-523 d$ & $16013-16569 / 1-588$ \\
\hline $43 \mathrm{~A}$ & M7b & 129223297 & $73150159199263315+\mathrm{C} 489$ & $16014-16569 / 1-559$ \\
\hline $43 \mathrm{~B}$ & M7b & 129223297 & $73150159199263315+C 489$ & $16017-16569 / 1-587$ \\
\hline $43 \mathrm{C}$ & M7b & 129223297 & $73150159199263315+C 489$ & $16014-16569 / 1-550$ \\
\hline $44 \mathrm{~A}$ & B5a & $140183 \mathrm{C} 189266 \mathrm{~A} 519$ & $73210263 \mathbf{3 0 9 + 2 C} 315+\mathrm{C} 522-523 \mathrm{~d}$ & $16014-16569 / 1-554$ \\
\hline $44 \mathrm{~B}$ & $\mathrm{~B} 5 \mathrm{a}$ & $140183 \mathrm{C} 189266 \mathrm{~A} 519$ & 73210263 309+C 310d $315+C$ 522-523d & $16012-16569 / 1-587$ \\
\hline $44 \mathrm{C}$ & $\mathrm{B} 5 \mathrm{a}$ & $140183 \mathrm{C} 189266 \mathrm{~A} 519$ & $73210263 \mathbf{3 0 9 + 2 C} 315+3 \mathrm{C}$ & $16018-16569 / 1-316$ \\
\hline $45 \mathrm{~A}$ & F1a & 129172304311519 & $73249 d 263315+C$ 522-523d & $16012-16569 / 1-585$ \\
\hline $45 \mathrm{~B}$ & F1a & 129172304311519 & $73249 \mathrm{~d} 263315+\mathrm{C} 522-523 \mathrm{~d}$ & $16012-16569 / 1-587$ \\
\hline $45 \mathrm{C}$ & F1a & 129172304311519 & $73249 d 263315+C$ 522-523d & $16013-16569 / 1-586$ \\
\hline $46 \mathrm{~A}$ & B6 & $093179182 \mathrm{C} 183 \mathrm{C} 189342$ & $73150263 \mathbf{3 0 9}+\mathbf{5 C} 522-523 d$ & $16016-16569 / 1-602$ \\
\hline $46 \mathrm{~B}$ & B6 & $093179182 \mathrm{C} 183 \mathrm{C} 189342$ & $73150263309+2 C$ 522-523d & $16016-16569 / 1-531$ \\
\hline $46 \mathrm{C}$ & B6 & $093179182 \mathrm{C} 183 \mathrm{C} 189342$ & $73150263 \mathbf{3 0 9 + 5 C} 522-523 \mathrm{~d}$ & $16013-16569 / 1-542$ \\
\hline $47 \mathrm{~A}$ & M7b & 129192223297 & $73150199263309+2 C 315+C 489522-523 d$ & $16012-16569 / 1-591$ \\
\hline $47 \mathrm{~B}$ & M7b & 129192223297 & $73150199263309+2 C 315+C$ & $16021-16569 / 1-329$ \\
\hline $47 \mathrm{C}$ & M7b & 129192223297 & $73150199263309+2$ C $315+C 489$ & $16021-16569 / 1-554$ \\
\hline $48 \mathrm{~A}$ & A & 223278290319519 & $73151152235263309+C$ 315+C 522-523d & $16013-16569 / 1-587$ \\
\hline $48 \mathrm{~B}$ & $\mathrm{~A}$ & 223278290319519 & $73151152235263309+C \quad 315+C \quad 522-523 d$ & $16013-16569 / 1-575$ \\
\hline $48 \mathrm{C}$ & A & 223278290319519 & $73151152235263309+\mathrm{C} 315+\mathrm{C} 522-523 \mathrm{~d}$ & $16013-16569 / 1-583$ \\
\hline $49 \mathrm{~A}$ & $\mathrm{~B} 5 \mathrm{a}$ & $140183 \mathrm{C} 189262266 \mathrm{~A} 519$ & $73210263315+C 522-523 d$ & $16017-16569 / 1-585$ \\
\hline $49 \mathrm{~B}$ & $\mathrm{~B} 5 \mathrm{a}$ & $140183 \mathrm{C} 189262266 \mathrm{~A} 519$ & $73210263315+C 522-523 d$ & $16013-16569 / 1-588$ \\
\hline $49 \mathrm{C}$ & $\mathrm{B} 5 \mathrm{a}$ & $140183 \mathrm{C} 189262266 \mathrm{~A} 519$ & $73210263315+C 522-523 d$ & $16009-16569 / 1-586$ \\
\hline $50 \mathrm{~A}$ & F1b & $183 \mathrm{C} 189323 \mathrm{~A} 249304311$ & $73249 \mathrm{~d} 263315+\mathrm{C} 522-523 \mathrm{~d}$ & $16018-16569 / 1-586$ \\
\hline $50 \mathrm{~B}$ & F1b & $183 \mathrm{C} 189232 \mathrm{~A} 249304311$ & $73249 \mathrm{~d} 263315+\mathrm{C} 522-523 \mathrm{~d}$ & $16013-16569 / 1-579$ \\
\hline $50 \mathrm{C}$ & F1b & $183 \mathrm{C} 189232 \mathrm{~A} 249304311$ & $73249 \mathrm{~d} 263315+\mathrm{C} 522-523 \mathrm{~d}$ & $16013-16569 / 1-573$ \\
\hline $51 \mathrm{~A}$ & $\mathrm{D}$ & 174223311320362 & $73152263309+\mathrm{C} 315+\mathrm{C} 489$ & $16012-16569 / 1-586$ \\
\hline $51 \mathrm{~B}$ & $\mathrm{D}$ & 174223293311320362 & $73152178263309+C \quad 315+C 489$ & $16009-16569 / 1-586$ \\
\hline $51 \mathrm{C}$ & $\mathrm{D}$ & 174223311320362 & $73152263309+\mathrm{C} 315+\mathrm{C} 489$ & $16013-16569 / 1-587$ \\
\hline $52 \mathrm{~A}$ & $\mathrm{~F}$ & 092A 093234291304 & $73249 d 263315+C$ 522-523d & $16013-16569 / 1-586$ \\
\hline $52 \mathrm{~B}$ & $\mathrm{~F}$ & 092A 093234291304 & $73249 \mathrm{~d} 263315+\mathrm{C} 522-523 d$ & $16014-16569 / 1-546$ \\
\hline $52 \mathrm{C}$ & $\mathrm{F}$ & 092A 093234291304 & $73249 \mathrm{~d} 263315+\mathrm{C} 522-523 \mathrm{~d}$ & $16013-16569 / 1-546$ \\
\hline $53 \mathrm{~A}$ & D4 & 129223278362 & $73263315+C 489$ & $16013-16569 / 1-543$ \\
\hline $53 \mathrm{~B}$ & D4 & 129223278362 & $73263315+C 489$ & $16013-16569 / 1-558$ \\
\hline $53 \mathrm{C}$ & D4 & 129223278362 & $73263315+C 489$ & $16013-16569 / 1-554$ \\
\hline $54 \mathrm{~A}$ & F1b'd & $185189266 \mathrm{G} 291304519$ & $73249 \mathrm{~d} 263315+\mathrm{C}$ & $16017-16569 / 1-585$ \\
\hline $54 \mathrm{~B}$ & F1b'd & $185189266 \mathrm{G} 291304519$ & $73249 d 263315+C$ & $16013-16569 / 1-384$ \\
\hline $54 \mathrm{C}$ & Flb'd & $185189266 \mathrm{G} 291304519$ & $73249 \mathrm{~d} 263315+\mathrm{C}$ & $16005-16569 / 1-588$ \\
\hline $55 \mathrm{~A}$ & F1a & 108129162172189304519 & $73195245249 \mathrm{~d} 263315+\mathrm{C} 522-523 d$ & $16013-16569 / 1-587$ \\
\hline $55 \mathrm{~B}$ & F1a & 108129162172189304519 & $73195245249 d 263315+C$ 522-523d 750 & $16013-16569 / 1-758$ \\
\hline $55 \mathrm{C}$ & F1a & 108129162172189304519 & $73195245249 d 263315+C$ 522-523d & $16014-16569 / 1-543$ \\
\hline $56 \mathrm{~A}$ & $\mathrm{~F} 2$ & 093203231291295304519 & $73195249 \mathrm{~d} 263309+\mathrm{C} 315+\mathrm{C}$ & $16012-16569 / 1-554$ \\
\hline $56 \mathrm{~B}$ & $\mathrm{~F} 2$ & 093203231291304519 & $73195249 \mathrm{~d} 263309+\mathrm{C} 315+\mathrm{C}$ & $16015-16569 / 1-591$ \\
\hline $56 \mathrm{C}$ & $\mathrm{F} 2$ & 093203231291295304519 & $73195249 \mathrm{~d} 263309+\mathrm{C} 315+\mathrm{C}$ & $16000-16569 / 1-620$ \\
\hline $57 \mathrm{~A}$ & A & 223260290319519 & $6473146195235263309+C$ 315+C 522-523d & $16021-16569 / 1-533$ \\
\hline $57 \mathrm{~B}$ & $\mathrm{~A}$ & 223260290319519 & $\begin{array}{l}6473146195235263309+ \\
\text { C } 315+\text { C } 522-523 d 663750\end{array}$ & $16014-16569 / 1-777$ \\
\hline $57 \mathrm{C}$ & A & 223260290319519 & $6473146195235263309+2 C \quad 315+C$ 522-523d & $16017-16569 / 1-541$ \\
\hline $58 \mathrm{~A}$ & $\mathrm{~B} 5 \mathrm{a}$ & $093140183 \mathrm{C} 189266$ A 519 & $73146198210263309+2$ C $315+C 522-523 d$ & $16016-16569 / 1-543$ \\
\hline $58 \mathrm{~B}$ & $\mathrm{~B} 5 \mathrm{a}$ & $093140183 \mathrm{C} 189266 \mathrm{~A} 519$ & $73146198210263309+2$ C $315+C 522-523 d$ & $16012-16569 / 1-542$ \\
\hline $58 \mathrm{C}$ & B5a & 093140183 C 189 266A 519 & $73146198210263309+2$ C $315+C$ 522-523d & $16013-16569 / 1-555$ \\
\hline $59 \mathrm{~A}$ & M9a1a & $223234248265 C 316362$ & $73153263309+\mathrm{C} 315+\mathrm{C} 489$ & $16014-16569 / 1-573$ \\
\hline $59 \mathrm{~B}$ & M9a1a & 223234248265 C 316362 & $73153263309+\mathrm{C} 315+\mathrm{C} 489$ & $16009-16569 / 1-543$ \\
\hline $59 \mathrm{C}$ & M9a1a & $223234248265 C 316362$ & $73153263309+\mathrm{C} 315+\mathrm{C} 489$ & $16011-16569 / 1-543$ \\
\hline $60 \mathrm{~A}$ & $\mathrm{C}$ & 223290298327519 & 73146152249 d $263315+C 489$ & $16013-16569 / 1-584$ \\
\hline $60 \mathrm{~B}$ & $\mathrm{C}$ & 223290298327519 & $73146152249 \mathrm{~d} 263315+\mathrm{C} 489$ & $16014-16569 / 1-584$ \\
\hline $60 \mathrm{C}$ & $\mathrm{C}$ & 223290298327519 & 73146152249 d $263315+C 489$ & $16013-16569 / 1-585$ \\
\hline $61 \mathrm{~A}$ & $\mathrm{~N} 9 \mathrm{a}$ & 189223257 A 261311 & $73150263309+2 \mathrm{C} 315+\mathrm{C}$ & $16016-16569 / 1-579$ \\
\hline $61 \mathrm{~B}$ & N9a & $189223257 A 261311$ & $73150263309+2 \mathrm{C} 315+\mathrm{C}$ & $16015-16569 / 1-582$ \\
\hline $61 \mathrm{C}$ & N9a & 189223257 A 261311 & $73150263309+2 \mathrm{C} 315+\mathrm{C}$ & $16014-16569 / 1-572$ \\
\hline $62 \mathrm{~A}$ & $\mathrm{~B} 5 \mathrm{~b} 2$ & $093111140182 \mathrm{C} 183 \mathrm{C} 189234243463519$ & $73103131 \quad 146199204263 \quad \mathbf{3 0 9}+3 \mathrm{C} 315+\mathrm{C}$ & $16017-16569 / 1-323$ \\
\hline $62 \mathrm{~B}$ & $\mathrm{~B} 5 \mathrm{~b} 2$ & $093111140182 C \quad 183 C \quad 189234243463519$ & $73103131146199204263 \mathbf{3 0 9 + 2 C} 315+C$ & $16012-16569 / 1-317$ \\
\hline
\end{tabular}

Continued on next page 


\begin{tabular}{|c|c|c|c|c|}
\hline Sample & Haplogroups & HVSI (16000+) & HVSII & Readable region \\
\hline $62 \mathrm{C}$ & $\mathrm{B} 5 \mathrm{~b} 2$ & $093111140182 \mathrm{C} 183 \mathrm{C} 189234243463519$ & 73103131146199204263 309+3C $315+C$ & $16011-16569 / 1-322$ \\
\hline $63 \mathrm{~A}$ & B4a & $182 \mathrm{C} 183 \mathrm{C} 186189217261360519$ & $73263315+C 522-523 d$ & $16013-16569 / 1-583$ \\
\hline $63 \mathrm{~B}$ & B4a & 182C 183C 186189217261360519 & $73263315+C \quad 522-523 d$ & $16009-16569 / 1-565$ \\
\hline $63 \mathrm{C}$ & B4a & 182C 183C 186189217261360519 & $73263315+C 522-523 d$ & $16009-16569 / 1-580$ \\
\hline $64 \mathrm{~A}$ & D4 & 129223278362 & $73263315+C 489$ & $16013-16569 / 1-580$ \\
\hline 64B & D4 & 129223278362 & $73263315+C 489$ & $16011-16569 / 1-585$ \\
\hline $64 \mathrm{C}$ & D4 & 129223278362 & $73263315+C 489$ & $16011-16569 / 1-565$ \\
\hline $65 \mathrm{~A}$ & $\mathrm{D} 4 \mathrm{~b}$ & 287319362390 & $73263315+C$ 420h $431489522-523 d$ & $16007-16569 / 1-587$ \\
\hline $65 \mathrm{~B}$ & $\mathrm{D} 4 \mathrm{~b}$ & 287319362390 & 73263 315+C 420431489 522-523d & $16016-16569 / 1-585$ \\
\hline $65 \mathrm{C}$ & $\mathrm{D} 4 \mathrm{~b}$ & 287319362390 & $73263315+$ +C 420h $431489522-523 d$ & $16013-16569 / 1-584$ \\
\hline $66 \mathrm{~A}$ & B4 & $182 \mathrm{C} 183 \mathrm{C} 189217240261$ & $73263309+$ C $315+2$ C $522-523 d$ & $16013-16569 / 1-554$ \\
\hline $66 \mathrm{~B}$ & B4 & $182 \mathrm{C} 183 \mathrm{C} 189217240261$ & $73263309+2$ C $315+C 522-523 d$ & $16017-16569 / 1-553$ \\
\hline $66 \mathrm{C}$ & B4 & $182 \mathrm{C} 183 \mathrm{C} 189217240261$ & $73263309+2$ C $315+C 522-523 d$ & $16016-16569 / 1-543$ \\
\hline $67 \mathrm{~A}$ & Z & 172185189223260298362519 & 73151152 249d $263315+C 489$ & $16014-16569 / 1-587$ \\
\hline 67B & Z & 172185223260298519 & 73151152 249d $263315+C 489$ & $16013-16569 / 1-583$ \\
\hline $67 \mathrm{C}$ & $\mathrm{Z}$ & 172185189223260298362519 & $73151152249 d 263315+C 489$ & $16013-16569 / 1-585$ \\
\hline $68 \mathrm{~A}$ & D4 & 223256311362519 & $73200263309+C$ 315+C $489522-523 d$ & $16021-16569 / 1-577$ \\
\hline $68 \mathrm{~B}$ & D4 & 223256311362519 & $73200263309+C$ 315+C $489522-523 d$ & $16013-16569 / 1-584$ \\
\hline $68 \mathrm{C}$ & D4 & 223256311362519 & $73200263309+C$ 315+C $489522-523 d$ & $16013-16569 / 1-585$ \\
\hline $69 \mathrm{~A}$ & $\mathrm{~F} 2$ & 124167203304318519 & $73249 d 263315+C$ & $16013-16569 / 1-560$ \\
\hline $69 \mathrm{~B}$ & $\mathrm{~F} 2$ & 124167203304318519 & 73249 d $263315+C$ & $16016-16569 / 1-585$ \\
\hline $69 \mathrm{C}$ & $\mathrm{F} 2$ & 124167203304318519 & 73 249d $263315+C$ & $16009-16569 / 1-573$ \\
\hline $70 \mathrm{~A}$ & B5a & $140187189256266 \mathrm{G} 519$ & $7393210263315+C 522-523 d$ & $16009-16569 / 1-585$ \\
\hline $70 \mathrm{~B}$ & B5a & $140187189256266 \mathrm{G} 519$ & $7393210263315+C$ 522-523d & $16013-16569 / 1-585$ \\
\hline $70 \mathrm{C}$ & B5a & $140187189256266 \mathrm{G} 519$ & $7393210263315+C 522-523 d$ & $16008-16569 / 1-571$ \\
\hline $71 \mathrm{~A}$ & D4 & 129223278362 & $73263315+2 \mathrm{C} 489523+\mathrm{CA}$ & $16013-16569 / 1-585$ \\
\hline $71 \mathrm{~B}$ & D4 & 129223278362 & $73263315+2 \mathrm{C} 489523+\mathrm{CA}$ & $16012-16569 / 1-585$ \\
\hline $71 \mathrm{C}$ & D4 & 129223278362 & $73263315+2 \mathrm{C} 489523+\mathrm{CA}$ & $16013-16569 / 1-562$ \\
\hline $72 \mathrm{~A}$ & B4a & $182 \mathrm{C} 183 \mathrm{C} 189217261$ & $73200263 \mathbf{3 0 9 + 2 C} 522-523 d$ & $16016-16569 / 1-543$ \\
\hline $72 \mathrm{~B}$ & $\mathrm{~B} 4 \mathrm{a}$ & $182 \mathrm{C} 183 \mathrm{C} 189217261$ & 73200263 309+CCCCA 522-523d & $16016-16569 / 1-543$ \\
\hline $72 \mathrm{C}$ & $\mathrm{B} 4 \mathrm{a}$ & $182 \mathrm{C} 183 \mathrm{C} 189217261$ & $73200263309+2 C 522-523 d$ & $16008-16569 / 1-554$ \\
\hline $73 \mathrm{~A}$ & Z & 185223260297298 & $73152189207249 d 263309+2 C \quad 315+C 489$ & $16006-16569 / 1-567$ \\
\hline $73 \mathrm{~B}$ & $\mathrm{Z}$ & 185223260297298 & $73152189207249 d 263309+2$ C $315+C 489$ & $16012-16569 / 1-543$ \\
\hline $73 \mathrm{C}$ & $\mathrm{Z}$ & 185223260297298 & 73152189207249 d $263309+2$ C $315+C 489$ & $16012-16569 / 1-520$ \\
\hline $74 \mathrm{~A}$ & M31 & 093136223 & $73146152263315+C 489$ & $16010-16569 / 1-567$ \\
\hline $74 \mathrm{~B}$ & M31 & 093136223 & $73146152263315+C 489$ & $16006-16569 / 1-569$ \\
\hline $74 \mathrm{C}$ & M31 & 093136223 & $73146152263315+C 489$ & $16024-16569 / 1-588$ \\
\hline $75 \mathrm{~A}$ & B5b2 & $093182 \mathrm{C} 183 \mathrm{C} 189217243261519$ & $73146204263309+C 315+C$ 522-523d & $16014-16569 / 1-587$ \\
\hline $75 \mathrm{~B}$ & $\mathrm{~B} 5 \mathrm{~b} 2$ & $093182 \mathrm{C} 183 \mathrm{C} 189217243261519$ & $73146204263309+C 315+C$ 522-523d & $16013-16569 / 1-543$ \\
\hline $75 \mathrm{C}$ & $\mathrm{B} 5 \mathrm{~b} 2$ & $093182 \mathrm{C} 183 \mathrm{C} 189217243261519$ & $73146204263309+2 C \quad 315+C$ & $16012-16569 / 1-316$ \\
\hline $76 \mathrm{~A}$ & D5 & 189223362519 & $73150263309+2 \mathrm{C} 315+\mathrm{C} 456489523+\mathrm{CA}$ & $16017-16569 / 1-543$ \\
\hline $76 \mathrm{~B}$ & D5 & 189223362519 & $73150263309+2 \mathrm{C} 315+\mathrm{C} 456489523+\mathrm{CA}$ & $16012-16569 / 1-543$ \\
\hline $76 \mathrm{C}$ & D5 & 189223362519 & $73150263309+2 \mathrm{C} 315+\mathrm{C}$ & $16010-16569 / 1-316$ \\
\hline $77 \mathrm{~A}$ & B4a & $093182 \mathrm{C} 183 \mathrm{C} 189217261519$ & $73146263309+\mathrm{C} 315+\mathrm{C}$ & $16012-16569 / 1-318$ \\
\hline $77 \mathrm{~B}$ & $\mathrm{~B} 4 \mathrm{a}$ & $093182 \mathrm{C} 183 \mathrm{C} 189217261519$ & $73146263309+C$ 315+C 522-523d & $16012-16569 / 1-554$ \\
\hline $77 \mathrm{C}$ & $\mathrm{B} 4 \mathrm{a}$ & $093182 \mathrm{C} 183 \mathrm{C} 189217261519$ & $73146263309+C$ 315+C 522-523d & $16012-16569 / 1-550$ \\
\hline $78 \mathrm{~A}$ & R9 & 304362519 & $73263315+\mathrm{C}$ & $16007-16569 / 1-590$ \\
\hline $78 \mathrm{~B}$ & R9 & 304362519 & $73263315+\mathrm{C}$ & $16007-16569 / 1-587$ \\
\hline $78 \mathrm{C}$ & R9 & 304362519 & $73263315+C$ & $16015-16569 / 1-368$ \\
\hline $79 \mathrm{~A}$ & $\mathrm{~B} 4 \mathrm{a}$ & $140183 \mathrm{C} 189266 \mathrm{~A} 311519$ & $73210263315+C 522-523 d$ & $16008-16569 / 1-558$ \\
\hline $79 \mathrm{~B}$ & $\mathrm{~B} 4 \mathrm{a}$ & $140183 \mathrm{C} 189266 \mathrm{~A} 311519$ & $73210263315+C 522-523 d$ & $16010-16569 / 1-550$ \\
\hline $79 \mathrm{C}$ & $\mathrm{B} 4 \mathrm{a}$ & $140183 \mathrm{C} 189266 \mathrm{~A} 311519$ & $73210263315+C 522-523 d$ & $16009-16569 / 1-542$ \\
\hline
\end{tabular}

As shown in Table 2, the frequencies of mtDNA haplogroups $\mathrm{F}^{*}$ and $\mathrm{G}^{*}$ showed significant differences between the lung cancer and control group, which indicated the possible associations between mtDNA haplogroups $\mathrm{F}^{*}$ and $\mathrm{G}^{*}$ and lung cancer groups from Yunnan, Southwest of China (at the level of $\mathrm{P}<0.05$ ). 


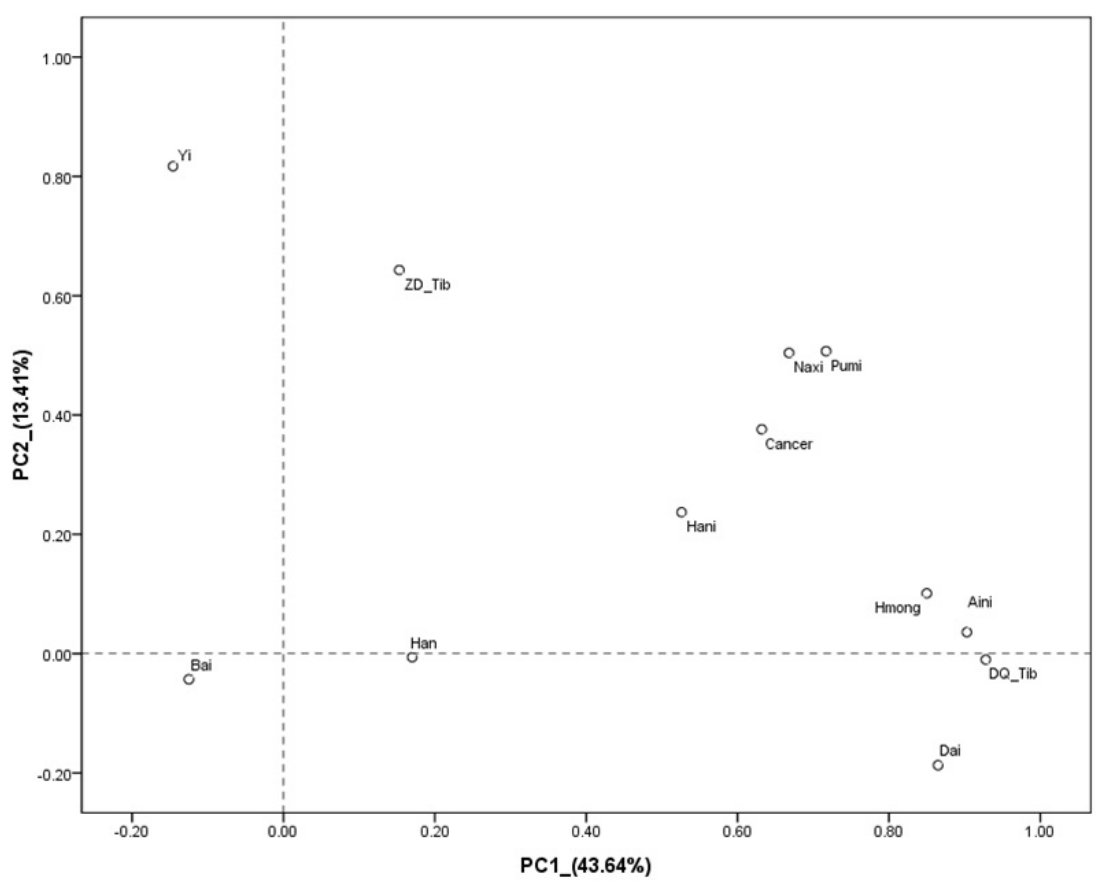

Figure 1. Principle component analysis (PCA) of the populations under study. ZD_Tib = Tibetan group from Zhongdian region of Yunnan Province; DQ_Tib = Tibetan group from Diqing region of Yunnan Province.

\begin{tabular}{|c|c|c|c|c|c|c|}
\hline Haplogroups & Lung cancer $(\mathrm{N})$ & Control (N) & $\chi^{2}$ & $P$ value & OR & $95 \%$ CI \\
\hline A & 8 & 34 & 1.011 & 0.315 & 1.151 & $0.672-3.396$ \\
\hline B & 1 & 5 & 0.039 & 0.594 & 1.244 & $0.143-10.787$ \\
\hline B4 & 10 & 47 & 0.71 & 0.4 & 1.366 & $0.660-2.829$ \\
\hline B5 & 6 & 27 & 0.541 & 0.439 & 1.409 & $0.563-3.351$ \\
\hline $\mathrm{C}$ & 3 & 27 & 0.4 & 0.786 & 0.677 & $0.200-2.286$ \\
\hline D & 4 & 33 & 0.313 & 0.576 & 0.739 & $0.254-2.145$ \\
\hline D4 & 9 & 49 & 0.144 & 0.704 & 1.157 & $0.544-2.460$ \\
\hline D5 & 3 & 19 & 0.001 & 1 & 0.979 & $0.283-3.387$ \\
\hline $\mathrm{F}$ & 3 & 3 & 6.651 & 0.038 & 6.408 & $1.270-32.330$ \\
\hline F1 & 10 & 51 & 0.36 & 0.549 & 1.248 & $0.605-2.573$ \\
\hline $\mathrm{F} 2$ & 2 & 12 & 0.002 & 1 & 1.035 & $0.227-4.712$ \\
\hline $\mathrm{G}$ & 1 & 37 & 4.312 & 0.038 & 0.157 & $0.021-1.161$ \\
\hline M & 3 & 14 & 0.208 & 0.718 & 1.342 & $0.377-40478$ \\
\hline M7 & 4 & 35 & 0.461 & 0.497 & 0.693 & $0.240-2.007$ \\
\hline M9 & 1 & 18 & 1.222 & 0.496 & 0.336 & $0.044-2.554$ \\
\hline N9 & 3 & 4 & 4.976 & 0.06 & 4.976 & $1.053-21.849$ \\
\hline $\mathrm{R}$ & 2 & 2 & 4.395 & 0.095 & 6.338 & $0.880-45.657$ \\
\hline R11 & 1 & 5 & 0.039 & 0.054 & 1.244 & $0.143-10.787$ \\
\hline R9 & 2 & 7 & 0.532 & 0.362 & 1.792 & $0.366-8.786$ \\
\hline Z & 3 & 6 & 2.893 & 0.116 & 3.184 & $0.780-13.001$ \\
\hline
\end{tabular}

*P value was calculated by the Pearson chi-square test at the level of $\mathrm{P}<0.05$; the Fisher exact test was used when haplogroups were expected to contain fewer than five individuals. 
Furthermore, as shown in our previous study, a large number of the identified somatic mutations among the patients with lung cancer from Yunnan, Southwest China, were detected by sequencing the entire mtDNA genome (Fang et al., 2013) and the mtDNA D310 region (Chen et al., 2014). In view of the relatively higher somatic mutation rate in the whole mtDNA D-loop (Yu 2012), and the relatively small sample sizes (Fang et al., 2013) and limited information obtained from our previous studies, which only analyzed the sequence variations within the mtDNA D310 region (Chen et al., 2014), we therefore sequenced and analyzed the complete mtDNA control region (except for the polymorphisms at D310) of 79 patients with lung cancer from Yunnan, Southwest China. Somatic mutations were detected by comparison of the sequences between tumor, matched normal tissues, and blood from the same patients, with the aim to provide more information toward better understanding the potential role of a given somatic mutation in tumorigenesis. As listed in Table 1, a total of eight somatic mutations were detected among six patients with lung cancer, including mutations at positions 16,093, 16,189, 16,362, 16,293, and 16,295 at HVSI, and mutations 178, 294 and 420 at HVSII/III. As shown in Figure 2, the authenticity of the eight somatic mutations was verified using the strategy described in our previous study (Fang et al., 2013; Chen et al., 2014). Incorporating the polymorphisms at D310 as reported in our previous study (Chen et al., 2014), a total of 20 individuals with somatic mutations were detected among 79 patients with lung cancer from Yunnan, Southwest China, which accounted for $24.05 \%$ (19/79) of the patients with lung cancer examined. By further analyzing these somatic mutations, we found that heterogeneity was the predominant characteristic of somatic mutations, in line with our previous results (Fang et al., 2013; Chen et al., 2014).
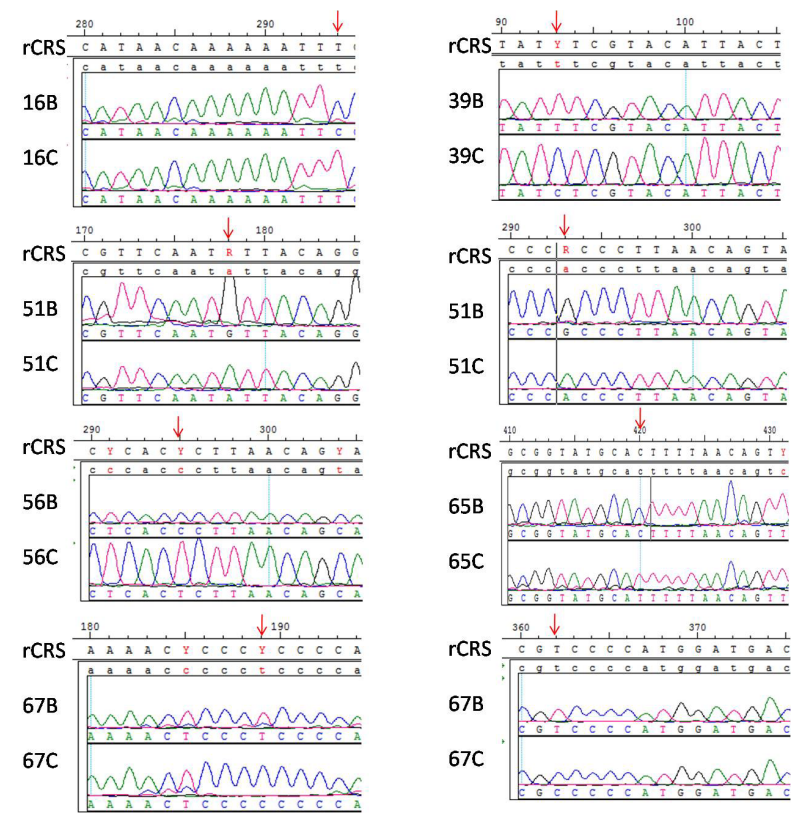

Figure 2. Somatic mtDNA mutations detected at the mtDNA control region in 79 Chinese patients with lung cancer. Sequencing results of the lung cancer tissue and distant normal tissue from the same patient are shown; sites of mutation are marked with red arrows. Mutations were identified by comparison with the revised Cambridge reference sequence (rCRS). B: mtDNA sequence from the primary tumor sample; C: mtDNA sequence from peripheral blood from the same patient. 


\section{DISCUSSION}

By extensively analyzing the mtDNA variations in 237 samples from 79 patients with lung cancer from Yunnan, we found that the frequency of East Asian and Southeast Asian prevalent haplogroups $(50.63 \%, 40 / 79)$ was higher than that of northern East Asian prevalent haplogroups $(44.30 \%, 35 / 79)$ of the patients. These haplogroup distributions were in line with those of healthy control groups from Yunnan Province (Yao et al., 2002b; Wen et al., 2004b), which may be a result of the admixture of autochthonous components from both northern and southern populations.

A total of 20 somatic mutations were detected among 19 of 79 patients with lung cancer from Yunnan, which implied the mtDNA somatic mutations confer genetic susceptibility to lung cancer in patients from Yunnan, Southwest China, and heterogeneity was the major characteristic of the 20 somatic mutations. The mtDNA D-loop is important for regulation of mitochondrial genome replication and expression. Therefore, the relatively elevated somatic mutation rates in the patients with lung cancer from Yunnan Province, Southwest China, suggested that the somatic mutations in these patients might affect the crucial mitochondrial function in the electron transport chain, which might in turn cause a high release of reactive oxygen species and concomitant nuclear genome damage as well as cancer initiation and promotion (Shigenaga et al., 1994; Li et al., 2012; Yu, 2012).

In summary, to test whether maternal background or mtDNA somatic mutations played a crucial role in Chinese patients with lung cancer, both the germline and somatic mutations of the mtDNA D-loop region were analyzed in 237 samples from 79 Chinese patients with lung cancer. The PCA and statistical analysis rejected the likelihood of population stratification, and further statistical analysis supported the existence of associations between mtDNA haplogroups $\mathrm{F}^{*}$ and $\mathrm{G}^{*}$ and the Chinese lung cancer group. Furthermore, a higher frequency of somatic mutations (25.32\%) in the mtDNA D-loop was detected among Chinese patients with lung cancer, which indicated that the somatic mutations might play crucial roles in the initiation and promotion of lung cancer. Our results suggest that mitochondrial DNA haplogroups and somatic mutations confer genetic susceptibility to lung cancer in patients from Southwest China, and that the somatic mtDNA mutations in the D-loop can serve as a potential biomarker for clinical utility.

\section{ACKNOWLEDGMENTS}

We are grateful to all the voluntary donors for participating in the project. Research supported by grants from the Nature Sciences Foundation of Yunnan Provincial Science and Technology Department (\#2012CA002 and \#2012HB030).

\section{REFERENCES}

Alhomidi MA, Vedicherla B, Movva S, Rao PK, et al. (2013). Mitochondrial D310 instability in Asian Indian breast cancer patients. Tumour Biol. 34: 2427-2432.

Anderson S, Bankier AT, Barrell BG, Bruijn MH, et al. (1981). Sequence and organization of the human mitochondrial genome. Nature 290: 457-465.

Andrews RM, Kubacka I, Chinnery PF, Lightowlers RN, et al. (1999). Reanalysis and revision of the Cambridge reference sequence for human mitochondrial DNA. Nat. Genet. 23: 147. 
Biffi A, Anderson CD, Nalls MA, Rahman R, et al. (2010). Principal-component analysis for assessment of population stratification in mitochondrial medical genetics. Am. J. Hum. Genet. 86: 904-917.

Brandon M, BaldiP and Wallace DC (2006). Mitochondrial mutations in cancer. Oncogene 25: 4647-4662.

Cao C, Zhang YM, Wang R, Sun SF, et al. (2011). Excision repair cross complementation group 1 polymorphisms and lung cancer risk: a meta-analysis. Chin. Med. J. 124: 2203-2208.

Chatterjee A, Mambo E and Sidransky D (2006). Mitochondrial DNA mutations in human cancer. Oncogene 25: 4663-4674.

Chen XZ, Fang Y, Shi YH, Cui JH, et al. (2014). Mitochondrial D310 instability in Chinese lung cancer patients. Mitochondrial DNA 10: 1-4. Doi: 10.3109/19401736.19402014.19936426.

Fang Y, Huang J, Zhang J, Wang J, et al. (2013). Detecting the somatic mutations spectrum of Chinese lung cancer by analyzing the whole mitochondrial DNA genomes. Mitochondrial DNA Sept. 6. [Epub ahead of print]. Doi: $10.3109 / 19401736.2013 .823168$.

Ferlay J, Shin HR, Bray F, Forman D, et al. (2010). Estimates of worldwide burden of cancer in 2008: GLOBOCAN 2008. Int. J. Cancer 127: 2893-2917.

Fuku N, Park KS, Yamada Y, Nishigaki Y, et al. (2007). Mitochondrial haplogroup N9a confers resistance against type 2 diabetes in Asians. Am. J. Hum. Genet. 80: 407-415.

Hung WY, Wu CW, Yin PH, Chang CJ, et al. (2010). Somatic mutations in mitochondrial genome and their potential roles in the progression of human gastric cancer. Biochim. Biophys. Acta 1800: 264-270.

Ji Y, Zhang AM, Jia XY, Zhang YP, et al. (2008). Mitochondrial DNA haplogroups M7b1'2 and M8a affect clinical expression of Leber hereditary optic neuropathy in Chinese families with the $\mathrm{m} .11778 \mathrm{G} \rightarrow$ a mutation. Am. J. Hum. Genet. 83: 760-768.

Jin X, Zhang J, Gao Y, Ding K, et al. (2007). Relationship between mitochondrial DNA mutations and clinical characteristics in human lung cancer. Mitochondrion 7: 347-353.

Kong QP, Sun C, Wang HW, Zhao M, et al. (2011). Large-scale mtDNA screening reveals a surprising matrilineal complexity in East Asia and its implications to the peopling of the region. Mol. Biol. Evol. 28: 513-522.

Kumimoto H, Yamane Y, Nishimoto Y, Fukami H, et al. (2004). Frequent somatic mutations of mitochondrial DNA in esophageal squamous cell carcinoma. Int. J. Cancer 108: 228-231.

Li H, Liu D, Lu J and Bai Y (2012). Physiology and pathophysiology of mitochondrial DNA. Adv. Exp. Med. Biol. 942: 39-51.

Mishmar D, Ruiz-Pesini E, Golik P, Macaulay V, et al. (2003). Natural selection shaped regional mtDNA variation in humans. Proc. Natl. Acad. Sci. U. S. A. 100: 171-176.

Ruiz-Pesini E, Mishmar D, Brandon M, Procaccio V, et al. (2004). Effects of purifying and adaptive selection on regional variation in human mtDNA. Science 303: 223-226.

Sharma H, Singh A, Sharma C, Jain SK, et al. (2005). Mutations in the mitochondrial DNA D-loop region are frequent in cervical cancer. Cancer Cell Int. 5: 34.

Shigenaga MK, Hagen TM and Ames BN (1994). Oxidative damage and mitochondrial decay in aging. Proc. Natl. Acad. Sci. U. S. A. 91: 10771-10778.

van Oven M and Kayser M (2009). Updated comprehensive phylogenetic tree of global human mitochondrial DNA variation. Hum. Mutat. 30: e386-394.

Wallace DC (1995). Mitochondrial DNA variation in human evolution, degenerative disease, andaging. Am. J. Hum. Genet. 57: 201-223.

Wallace DC (2005). A mitochondrial paradigm of metabolic and degenerative diseases, aging, and cancer: a dawn for evolutionary medicine. Annu. Rev. Genet. 39: 359-407.

Wallace DC, Brown MD and Lott MT (1999). Mitochondrial DNA variation in human evolution and disease. Gene 238: 211-230.

Wang CY and Zhao ZB (2011). Somatic mtDNA mutations in lung tissues of pesticide-exposed fruitgrowers. Toxicology 291: 51-55.

Wang CY, Wang HW, Yao YG, Kong QP, et al. (2007). Somatic mutations of mitochondrial genome in early stage breast cancer. Int. J. Cancer 121: 1253-1256.

Wen B, Li H, Lu D, Song X, et al. (2004a). Genetic evidence supports demic diffusion of Han culture. Nature 431: 302 305.

Wen B, Xie X, Gao S, Li H, et al. (2004b). Analyses of genetic structure of Tibeto-Burman populations reveals sex-biased admixture in southern Tibeto-Burmans. Am. J. Hum. Genet. 74: 856-865.

Wen B, Li H, Gao S, Mao X, et al. (2005). Genetic structure of Hmong-Mien speaking populations in East Asia as revealed by mtDNA lineages. Mol. Biol. Evol. 22: 725-734.

Williams SL, Mash DC, Zuchne S and Moraes CT (2013). Somatic mtDNA mutation spectra in the aging human putamen. PLoS Genet. 9: e1003990. 
Yakes FM and Van Houten B (1997). Mitochondrial DNA damage is more extensive and persistslonger than nuclear DNA damage in human cells following oxidative stress. Proc. Natl. Acad. Sci. U. S. A. 94: 514-519.

Yang L, Yang G, Zhou M, Smith M, et al. (2009). Body mass index and mortality from lung cancer in smokers and nonsmokers: a nationally representative prospective study of 220,000 men in China. Int. J. Cancer 125: 2136-2143.

Yang Ai SS, Hsu K, Herbert C, Cheng Z, et al. (2013). Mitochondrial DNA mutations in exhaled breath condensate of patients with lung cancer. Respir. Med. 107: 911-918.

Yao YG, Kong QP, Bandelt HJ, Kivisild T, et al. (2002a). Phylogeographic differentiation of mitochondrial DNA in Han Chinese. Am. J. Hum. Genet. 70: 635-651.

Yao YG, Nie L, Harpending H, Fu YX, et al. (2002b). Genetic relationship of Chinese ethnic populations revealed by mtDNA sequence diversity. Am. J. Phys. Anthropol. 118: 63-76.

Yu M (2012). Somatic mitochondrial DNA mutations in human cancers. Adv. Clin. Chem. 57: 99-138.

Zhao M, Kong QP, Wang HW, Peng MS, et al. (2009). Mitochondrial genome evidence reveals successful Late Paleolithic settlement on the Tibetan Plateau. Proc. Natl. Acad. Sci. U. S. A. 106: 21230-21235. 